\title{
Exploring communication of the HIV/AIDS health policy change to test-and- treat and its effect on policy implementation in selected public health facilities in Lusaka district, Zambia
}

Constance Simooya

University of Zambia

Adam Silumbwe ( $\sim$ adam.silumbwe@umu.se)

Centre for Research in Environmental Epidemiology: Instituto de Salud Global de Barcelona https://orcid.org/0000-0002-1905-5293

Hikabasa Halwindi

University of Zambia

Joseph Mumba Zulu

University of Zambia

Selestine Nzala

University of Zambia

Research

Keywords: Communication, HIV/AIDs, implementation, policy change, test and treat

Posted Date: February 15th, 2022

DOI: https://doi.org/10.21203/rs.3.rs-1314278/v1

License: (c) (i) This work is licensed under a Creative Commons Attribution 4.0 International License. Read Full License 


\section{Abstract \\ Background}

The World Health Organisation has promoted a shift towards the test and treat strategy to accelerate elimination of HIV/AIDS. Zambia adopted this policy change in 2017. However, communication of this policy change and its effect on policy implementation has not been documented. This study sought to explore the communication of the HIV/AIDS test and treat health policy change and how this affects practice in selected public health facilities in Lusaka district.

\section{Methods}

A qualitative case study was employed with purposeful sampling of policy makers, partners, National AIDS Council managers and frontline health providers in selected tertiary, secondary and primary-level health facilities in Lusaka District of Zambia. In total, 22 key informant interviews and 3 focus group discussion were conducted. Thematic inductive - deductive approach coding and data analysis were performed using NVivo pro 12 software.

\section{Results}

The results revealed that to communicate test and treat policy change; government relied on print media such as newspapers, IEC materials and electronic media through radios and television. The official channels of communication and feedback mechanisms for test and treat HIV health policy change were not clearly stipulated in the health facilities for reference. This created a lapse in communication which resulted in policy misinterpretation and resistance from both implementers and clients. The HIV policy changes reshaped health systems negatively as infrastructure was altered to accommodate increased service demand workload for HIV services and operational hours for HCWs. Further, policy makers seemed to be unprepared for the policy changes.

\section{Conclusion}

Effective health policy change communication with clear channels and open feedback mechanisms is vital for successful policy change implementation as it enhances understanding and subsequently adoption of the policy. Further, there is a need to enhance collaboration amongst policy makers, partners, implementers and the public in developing and applying communication strategies that facilitate quick adoption of health policy changes.

\section{Contributions To The Literature}

1. This study contributes to the limited literature on how communication of policy change affects policy implementation. Communication of policy change is critical in ensuring that policy intentions find the correct expressions in the health systems.

2. Effective health policy change communication with clear channels and open feedback mechanisms is vital for successful policy change implementation as it enhances understanding and subsequently adoption of the policy

3. There is a need to enhance collaboration amongst policy makers, partners, implementers and the public in developing and applying communication strategies that facilitate quick adoption of health policy changes

\section{Background}

Globally approximately 36.9 million people are living with HIV/AIDS [1]. Sub-Saharan Africa carries a disproportionate burden of HIV/AIDS, accounting for more than $70 \%$ of the global burden [2]. In Zambia, HIV prevalence is estimated to be $11 \%$ among adults while close to $15 \%$ of children are HIV positive. The HIV prevalence is higher among urban than rural residents (9.8\% vs 5.0\%) [3]. Further, there has been an increase in HIV prevalence among urban young men from $3.7 \%$ in $2001-2$ to $7.3 \%$ in 2013-14[4], and 9.4 million Zambians do not know their HIV status [5].

In tackling the HIV/AIDS infection, Zambia has adopted various HIV/AIDS policies over the years. These include the HIV/AIDS voluntary counselling and testing (VCT) strategy which was expanded to maternal health in an effort to achieve prevention of mother to child transmission (PMTCT) in 1999. In 2002, the government of Zambia created the National HIV/AIDS/STI/TB Council (NAC) whose mandate was to oversee HIV, sexually transmitted Infections and tuberculosis policies and programs. The creation of NAC saw the introduction of many HIV policies such as HIV work policy in all sectors; counselling and testing (C\&T) policy, and the HIV test post exposure prophylaxis in health facilities for exposed health workers and clients [6]. In 2013, diagnosis, counselling and testing (DCT) was introduced where all admitted patients were mandated to undergo HIV testing and counselling [7].

Changes to the HIV policy saw the mother to child transmission evolve from voluntary PMTCT to option A, B, compulsory PMTCT option B+ to now Elimination of mother to child transmission (EMTCT) [8]. In line with World Health Organization (WHO) call to zero rate new HIV infections and mortality, Zambia adopted the test-and-treat policy or the ' $90-90-90$ target' in 2017, a shift from passive voluntary counseling and testing. This policy entails that $90 \%$ of the people living with HIV know their HIV status; $90 \%$ of the people living with HIV who know their HIV status are accessing treatment; and $90 \%$ of people living with HIV who are receiving treatment have suppressed viral load. The target 90/90/90 aims to control HIV infections by the year 2020 and eliminate it by 2030 [9].Through a test and treat, if a person tests HIV-positive, he or she is immediately enrolled on HIV treatment. Before the advent of test and treat, people living with HIV had to wait for their CD4 count to drop to a particular level before starting treatment. Huge progress has been made since the adoption of the 
test and treat. For example, the proportion of ART initiation increased from 52\% in 2004-2006 to $97 \%$ in $2016-2017$ and time from testing to ART initiation reduced from a median of 17 days in 2004 to one day in 2016-2017among children living with HIV/AIDs[10].

Despite the progress in HIV/AIDS policy in Zambia over the years, HIV/AIDS still remains one the greatest threats to population health [3]. The changes in HIV/AIDS policy have come with some implementation successes and challenges similar to other low- and middle-income countries. Global influences by international funders have shaped the HIV/AIDS policy changes. Contextual factors such as social stigma and discrimination, traditional and religious beliefs, disease epidemiology, leadership, human resource, political and economic challenges have equally affected the success of HIV/AIDS policy changes[11]. Other notable implementation gaps for HIV/AIDs policies relate to retention on treatment, availability of nutritional supplements, support groups or isoniazid preventive therapy[12, 13].

While the above implementation gaps of HIV/AIDS policy changes have been widely documented, how these policy changes have been communicated has been understudied. Studies show that the manner in which health policies changes are introduced and communicated in a health system has potential to impact positively or negatively on implementation success [14]. Communication of HIV/AIDS policy changes is critical to ensure policy intentions find the correct expression in the health systems efforts to improve population health outcomes[15]. Health policy changes alter the normal functioning of health systems, hence the need for appropriate communication $[16,17]$. Understanding how HIV/AIDS policy changes are communicated, would therefore help enhance the quality of policy change implementation.

This study sought to explore the communication of the HIV/AIDS test-and-treat health policy change and how this affects practice in selected public health facilities in Lusaka district.

\section{Methods}

\section{Study Design}

A qualitative case study was undertaken. The case study was appropriate for this policy study because it allowed the researcher to interact with key stakeholders involved in health policy change communication and implementation. Further, it allowed for detailed exploration of HIV policy change communication processes and how this shaped the health system $[18,19]$. Case studies are most appropriate for policy studies. The study uses the consolidated criteria for reporting qualitative research (COREQ) guidelines (additional file).

\section{Study Site}

The study was conducted in Lusaka district the capital city of Zambia. It was purposefully selected due to the role it plays in policy leadership and oversight, as most high-level HIV policy institutions including the Ministry of Health (MoH), National Aids Council and other partner institutions are found in the capital. Policy changes are mostly decided in Lusaka and then communication all other peripheral cities, towns and villages. The health facility sites were selected based on recommendation from MoH policy makers. These were facilities that reported a high influx of HIV/AIDS clients on daily basis based on data submitted to $\mathrm{MoH}[20]$.

\section{Sampling criteria and participant recruitment}

Purposeful sampling method was employed to select participants relevant to the study who were at different levels of health care systems, with varied expertise in HIV/AIDS management which allowed to capture rich data from different perspectives. The health facility participants were purposefully sampled. Snowballing was also employed and helped to identify key participants in communication processes of test and treat policy. The policy makers were recruited using snowball sampling. With the cascade of hierarchy participants were selected to explore the communication policy process to identify bottlenecks and potential areas of improvement.

\section{Data collection}

The qualitative data were collected over a period of five months from November 2019 to March 2020. Phone calls were made to potential participants to arrange for face-to-face interview dates. Data were collected using recorders and notebooks respectively with consent from individual participants. To avoid any breach to privacy interviews were carried out in participants' usual official place of work privately. Data was stored in audio recorders; Laptop computers secured by password and note books safely in lockable cupboards in investigators 'office. To ensure confidentiality, all records were reviewed at the same sites under custody of principal investigator without moving them to other locations. The questions in the interview guides were phrased according to category of participants to bring out information on their roles about the change in HIV policy, communication, support and implementation. The interviews were conducted in English by the principal investigator (CS).

\section{Key informant interviews}

The key informants were policy makers from MoH; NAC managers; Lusaka District Health Office (LDHO) HIV/AIDS programme managers; partners; health facility managers from the primary; secondary and tertiary health care facilities involved in HIV/AIDS management and policy communication processes. A total of 22 key informant interview were held across Lusaka, with key state and non-state actors in HIV/AIDS policy change (table 1)

\section{Focus group discussions}

Three focus group discussions (FGD) with frontline healthcare professionals involved in health implementation of test and treat health policy change from the primary, secondary and tertiary health care facilities were conducted. The FGDs were conducted within private areas of frontline HCWs to allow freedom of 
expression away from their supervisors. The FGDs had 7-11 participants per session. The cadres comprised of clinicians and psychosocial counsellors specific to HIV/AIDS management. A cumulative number of 26 participants participated. The table below shows the level of health care facility, number of participants and their specialty (table 2).

\section{Data analysis}

The audio recorded interviews were transcribed using Express scribe v8.26 (NCH software, Canberra, Australia) and google docs (Google LLC, California and USA). Data from various sources were first read separately to develop themes and categories (table 3) [21]. The themes and categories centred on expressions, incidents, phrases and reactions during interviews, discussions and observations. All KIls and FGDs were separately compared and contrasted with each other. This was to help identify patterns, consensus, variations or contradictions in deliberating, frequency of phrases, emphasis, consistency in raising specific issues and answering the set objectives. The relative relevance of information based on emphasis by the study participants as these were at different levels of health care systems showed variations in policy communication. These informed the selection of broader theme categories for presentation in the study while eliciting ideas from literature review and the theoretical framework [22]. Data was then analyzed using the priori thematic themes. This type of approach was appropriate because certain concepts on HIV policies existed in current HIV services and literature. The focus was on standard communication of test and treat HIV health policy change; though other new ideas that emerged related to test and treat health policy change were included. Based on framework analysis and literature, inductive - deductive approach coding and analysis were performed manually by developing a medium for evolving categories and themes linked to the predefined ideas of the framework, conversations and experience (table 3) [23].

\section{Ethical considerations}

Approvals were sought from ethics committees; the University of Zambia Biomedical Research and Ethics Committee (UNZABREC) [reference number 2772019] and National Health Research Authority (NHRA). Permissions to conduct interviews in the intended health facilities were sought from the Provincial Health Offices, District Health Offices, individuals and health facilities managers. Voluntary written consents from individual participants were obtained.

\section{Results}

We present our findings on the communication of the test and treat all policy change in Zambia based on the policy makes and health provider perspectives. Emerging themes from the data included; communication strategy for HIV/AIDS policy change, implementation of policy change, effect of policy change on practice and acceptability of policy change. Although data were collected from various participant categories, no major differences in the discussions were noted and views specific to a particular participant category are noted within the manuscript.

\subsection{Communication strategy for HIV/AIDS policy change to test-and-treat}

\subsection{HIV/AIDS strategic framework for 2017 to 2021}

The policy makers mentioned that government had outlined a policy communication strategy that was operationalised through the HIV/AIDS strategic framework for 2017 to 2021. The strategic framework provides for coordination and management of the policy response to HIV/AIDS in Zambia. The coordination of the HIV/AIDS policy change takes place at national, provincial, district and community levels by aligning national priorities, expected outcomes and targets that all stakeholders should work towards. However, the frontline health providers revealed that the awareness strategies for HIV/AIDS policy change to test-and-treat-all that were put in place by the $\mathrm{MoH}$ through the strategic framework were inadequate. They narrated that the change to testand-treat policy took them by surprise, as there had been limited prior information. They felt that the policy change was abruptly introduced in the health systems without orientation.

"There is also a communication strategy, guided by HIV/AIDS strategic framework, on how each sector of the health system is going to be informed from the facilities, to community leaders to the people living with HIV to even those who would take care of those people living with HIV." (KII 02 Policy maker MoH 02)

"Even us health workers we were not ready for it. They just said, just go and test, so we had to put ourselves in their shoes, the clients; we had to explain that it was a government policy or program so we just had to do it without explaining its benefit." (FGD 03 Primary health facility participant)

\subsection{Communication channels of policy change}

The policy makers stated that to communicate policy, MoH generates a circular that cascades to the provincial health offices, district offices, tertiary secondary through to primary health care facilities and vice versa. However, the frontline health providers indicated that these processes seemed not to have been fully utilized when introducing the test-and-treat policy. Even when the above channels of communication were followed, the communication of policy changes delayed to reach implementers and the community. Some providers stated that verbal instructions via phone calls as well as informal messages about the change in policy to test-and-treat were given before written instructions were provided to the health facilities.

'So, policy ideally is communicated from the district, the district gets it from the province, province from the ministry. But with new technology now many are the times where you just see a WhatsApp message, oh! This is from the ministry but we always like to wait for official communication from the district before we act on anything..." (KII 07 Secondary health facility manager)

\subsection{The role of the media in communicating the policy change to test-and-treat-all}

\subsubsection{National television and radio stations}


The frontline HCWs mentioned that the media played an important role in communicating the test and treat policy to both the public and facilities. The test and treat policy change were first communicated to the public through the national television by the president of the republic of Zambia. It was reported that it was during the presidential pronouncement that some of the frontline HCWs got to know about the HIV test and treat policy. Following the presidential pronouncement many media houses such as print and radio amplified the sensitization of the test and treat policy. The health care providers indicated that since the president had to make this announcement on national television to reach the entire country, it signified the importance government attached to this particular policy change.

"I personally got it from TV that there is test and treat there was no communication from management, am sure most of us got it from TV..." (FGD 01, Tertiary health facility participant)

'Sensitization was done using TV and radios, but it's like many people did not understand what it meant. A lot of people we were still in denial despite being told, about that test and treat policy. But it took some time when it started it wasn't easy after sometime people came to understand and by now I can say it's only about $10 \%$ of people who are still refusing the test and treat' (KII 08 DHO manager 01)

\subsubsection{Information education and communication, and other print media}

The policy makers mentioned that newspapers, brochures, and posters were used to communicate the test and treat policy change to the public. However, the frontline health care providers felt that distribution of print media did not reach certain populations as some people in most remote areas were unable to read and had no access. They thought using print media to communicate the test and treat policy change was ineffective for certain sections of society. For instance, few people from the community could afford to buy newspapers. The majority of the people who read newspapers are senior government officials as they are routinely provided to them as part of their office privilege. The providers also mentioned that late delivery of print media affected real time communication of the policy change. It was suggested that use of online print media especially via social media platforms like Facebook, Instagram, WhatsApp, and messenger should have been used as it could be accessed by those with smart phones as they said:

"I can't say ah no we were not involved in developing IEC materials, again what comes first is English posters, these our people cannot read, even local language sometimes it becomes political which language to hang up. The message on pictures sometimes needs to be explained. Even me I think I don't know if you ask me details on posters..." (KII 12 primary health facility manager 02)

"The social media television, radios or WhatsApp, Facebook or SMS all this is very effective, even young people like these online issues there is also a lot of gossip so they can get the information using distractors..." (FGD 01 Tertiary health facility participant)

\subsection{Implementation of test and treat HIV policy change}

\subsection{Historical context and knowledge and history of HIV/AIDS health policy changes}

All the participants were cognizant of HIV/AIDS health policy changes in Zambia over the years. Policy makers and health facility managers were more knowledgeable on history of HIV policies; while those from primary and secondary levels were not keen about when and why polices were changed or phased out. Though not in order, participants were able to mention many previous HIV policies. The commonly cited policies were DCT, option B+ under maternal health and VCT which they felt had was present in all policies due to its emphasis on the counselling aspect. The participants in all the health facilities felt that they were still actively using the VCT but only applied it differently. This is because counselling was being offered to all clients as they provided test and treat. The points of services were also still being referred to as VCT rooms.

"Remember these things have been happening for a long time, there has been testing but it become now compulsory that everybody who come must be tested and tested counselled though it started with VCT and DCT..."(KII 04 Tertiary health facility manager 01)

"VCT is still there some people still walk into a facility on their own position to offer to have a test yeah. You can differentiate test and treat really to..., the only difference is that there is no gap now ok, no waiting for cd4 count to drop that's the major difference with test and treat" (KII 05 NAC manager 01 )

\subsection{Stakeholder engagement in test and treat health policy change}

The facility managers described that the government engaged various stakeholders such as public services organizations, companies and civil organizations to encourage people to take-up HIV tests including access to self-testing. Partners who were running different HIV programs in the health facilities were also engaged to support the test-and-treat policy. These partners such as PEPFAR, CDC played a vital role in providing resources to advertise and explain the HIV policy change to test-and-treat. Similarly, community-based structures through local health committees were engaged to support the policy change to test-andtreat. However, the participants felt that the engagement was mostly top-down, and not much room was provided to improve this relationship given the rapid change in policy and short time to implementation. A frontline HCW and health facility manager said:

"I would really say they need to look at this ... when a policy first of all at formulation level they should really involve the grass root, implementers of this policy. As the policy is being formulated, find that it is passed and it has to be implemented again let the orientation begin with the key implementers" (KII 07 Secondary health facility manager)

\subsection{Healthcare worker training for implementation of test-and-treat all policy}

Both the health facility managers and frontline providers indicated that there was some training that was intiated for those providing HIV care in the facilities. These training were undertaken in form of orientations given to a few selected health staff from tertiary and secondary health facilities. Some facilities were 
selected as pilot centres for test-and-treat policy before national wide scale-up. The facilities provided valuable lessons before the policy was expanded to other primary facilities. However, some participants felt that not much was head about this pilot, as it seemed to only cover a few health facilities. Key lessons were not communicated to the providers in other facilities. Further, the policy makers agreed that information in the health facilities at all levels of care concerning this policy was not uniform due to different levels of individual service provision and staff capacity. The tertiary levels had in most cases highly specialised officers due to required service standards including concerning HIV management and vice versa for lower levels of health facilities.

"...So, throughout the country Yes; the test and treat policy is it being practiced, ah the variance in implementation may be there; what I mean is we have guidelines on how the test and treat may be implemented but, in some areas, it's is a bit difficult to implement in such a way because they may not be receiving drugs directly from MSL (medical stores for life); they may not have lab facilities right there but in essence the test and treat is implemented everywhere...." (KII 02 Policy maker $\mathrm{MoH}$ 02)

\subsection{Materials and resources to support test and treat HIV health policy change}

The facility managers and providers explained that there was a differential support in terms of resources depending on the level of the facility as well as the volume of patients that were being attended to. However, most of the participants felt that majority of the health facilities did not receive the adequate supplies and commodities to support the scale-up of the test-and-treat policy. They stated that this resulted in many facilities experiencing stock-outs of key supplies for the policy at the time of scale-up. When the policy was pronounced, the front-line providers indicated that they struggled with resources for initiating HIV services starting with basic medical utilities such as testing kits and baseline investigations. Further, the providers reported that the primary health care facilities which were usually found in the slums with high volume populations more material supplies to be able to provide the services for testand-treat. A primary health facility manager said:

"I would be honest to say I don't know when the formulation began and whether maybe when the formulation began, we were or I personally was not in the district then, maybe meetings took place and what, but... from my knowledge I may be wrong I don't think we were involved We were not supported ........no, we were not supported. I can say for test and treat the key things we were struggling with even now things like urine stick, ok you want to have at least that dip stick you need as you are initiating the client..." (KII 07 Secondary health facility manager)

". So now the Ministry of Healthsupplied us with more testing kits, through our partners like CDC and CIRDZ; they employed more peers as treatment supporters also counsellors. So, we received more Counsellors, more testing kits; yes, so we were able to do all the activities." (KII 11 Primary health facility manager 01)

\subsection{Financing test and treat HIV health policy change}

The policy makers narrated that government had committed funding to the implementation of the test-and-treat policy change. However, the felt committed funds were still inadequate to carter for scale-up. For this reason, all participants indicated that funding from the partners was critical to sustain some of the policy changes in terms of service provision to HIV patients and management of care. The policy makers narrated that many partners were supporting the test and treat policy in community-based HIV programs. They increased funding and even funded staff to do part-time work to offset increased workload in selected high-volume primary level health facilities. However, both policy makers and health providers felt that having most funding for HIV services in the hands of donors was unsustainable given that this was a lifelong disease and it bordered on the economic development of the country. The partner support also included recruitment of staff at the health facilities, remuneration of staff, providing incentives, availing resources for policy monitoring and evaluation.

"... The ministry invested in this policy a lot. And partners came on board and we are grateful to this..." (KII 15 Policy maker MoH 03)

"... you know the country is divided into two parts, the northern region has six provinces: Copperbelt; central, north-western; Luapula; Muchinga and northern, these are supported by USAID. Then the others Lusaka, eastern, western and southern are supported by CDC. And each of these; they give funds to different organisations in these provinces, and thefunds they receive these organisations they are supposed to support the facilities under their care..." (KII 03 HIV partners - manager)

\subsection{Changes brought about by the HIV policy change to test-and-treat}

\subsection{Human resources and infrastructure adjustment}

The frontline HCWs and managers described how the test-and-treat policy silently changed the operations of the health systems from the primary, secondary and tertiary levels of health care. They explained that some of the changes brought about by this policy change included demand for human resource, operationalization; infrastructure physical readjustment of utilization of space in some facilities to accommodate the expanded services for counselling and screening; change in work schedules; increased working hours; patient interactions skills and service provision to patients just to mention a few. However, the policy makers mentioned that they did not anticipate an overhaul operation of the health systems due to the HIV policy change but expected services to run as usual. A policy maker said:

"... It changed the way we provide services because it meant that services were now tailored towards addressing that policy; but it was not easy on the ground because the staff and buildings remained the same, so workload increased because as a clinician as someone is attending to clients when you see that there is positive you have to shift certain things in the facility so that they receive the treatment there and then, and it was difficult...." (KII $06 \mathrm{DHO}$ manager)

\subsection{Acceptability of test and treat HIV health policy}

\subsection{Accepatability of policy among health providers}


Both the facility managers and frontline providers explained that the test and treat policy was widely accepted among healthcare workers as a long-awaited policy which had made HIV service provision easier. This is because they felt it contributed to reducing ill health of clients with advanced HIV. They thought early testing and treating of patients, as advocated for in the policy test-and-treat policy entailed putting the patients in better position to avoid opportunistic infections and mortality. Being able to manage HIV in its early stages meant that patient visitations to the facilities would be reduced, but also address congested HIV service infrastructure. The screening and checking procedures were made simple helping them to make accurate diagnoses after the HIV test results were known. A frontline HCW from a District Health Office said:

"... Now we benefited because it helped us by finding out what is disturbing this patient and you put the patient on the correct drugs and the two weeks later the patient recovers; patients who could have been on the ward for a long time, in and out but once you put someone on correct medicines 2 weeks later, they are fit..." (KII 08 DHO HIV program manager 02)

\subsection{Sense of ownership of HIV/AIDS policy change}

The health facility managers and frontline providers mentioned that they felt little sense of ownership of HIV/AIDS policy change to test-and-treat. They saw this HIV/AIDS policy change as more of an agenda of the ministry; hence they did not feel responsible for its successful implementation. Further, the health facility managers reported that policy makers from the $\mathrm{MoH}$ and partners seemed to have taken it for granted that health facility personnel would perform and implement the new HIV policy change to test-and-treat with minimal supervision making some individual providers to be hesitant about making certain decisions. The lack of institutional changes to support policy change such as restructuring human resource operations to accommodate and sustain policy change made local capacity to own the policy challenging. Furthermore, presence of various partner organisation pushing the policy change agenda also made the providers to question ownership and the intentions of the policy. One manager had this to say:

“... to implement anything that is new you must supervise, if you don't supervise many policies fall through but if you don't it will not work, if you supervise It will work... first of all policy must be mentored then, number two it must be explained, and then must be implemented and implementation involves supervision, supervision especially when you are just introducing something new..." (KII 04 Tertiary health facility manager 01)

\subsection{Policy change resistance from the community members}

Both the facility managers and frontline providers explained that when the test-and-treat policy was first rolled out to the public, many community members thought that it was just imposed on them and so they resisted it. They narrated that they had to spend a lot of time negotiating to convince the clients seeking health services to accept being tested-and-treated. The policy makers on the other hand seemed to have ignored the possible resistance to the test-and-treat policy from public. They seemed to have taken it for granted that somehow community members would appreciate the value of this policy given its benefits. Further, the policy makers seemed to have focused more on the statistics and targeted achievements of the test and treat policy. One policy maker said:

"...It was aimed at curative that all people found positive should be given ARVs for free, even testing is free and preventive level because we know those on HIV medications, we presumed would stop spreading the infection if they comply with treatment. From the numbers the policy is doing wonders a lot of people are on drugs now...." (KII 15 Policy maker MoH 03)

While an implementer had this to say:

"...so, it was difficult and it is still difficult. We are struggling with the issue of retention into care; but are fighting hard by continuing with sensitisation, community peers who follow them and to just explain to them on the benefits of being on medication. But I cannot lie we are losing people on medication especially those we put on medication during the period of test and treat because they were not ready..." (KI 08 DHO HIV Programme Manager 01 )

\section{Discussion}

The current study explored the communication of the HIV/AIDS test and treat health policy change and how this affected practice in selected public health facilities in Lusaka district. The results revealed that to communicate test and treat policy; government relied on print media such as newspapers, IEC materials and electronic media through radios and television which made very little impact to implementers and users. The official channels of communication and feedback mechanisms for test and treat HIV health policy change were not documented in health facilities for reference. This created a lapse in communication which resulted in policy misinterpretation and resistance from both users and implementers. The policy changes reshaped health systems physically as infrastructures were altered to accommodate increased service demand and operations that increased workload for HCWs.

We found that policy makers, partners, NAC managers and tertiary health facility managers had a better understanding of various HIV communication and implementation strategies for test and treat health policy change. However, the frontline HCWs were not aware of why or when HIV policies were frequently revised. The HCWs were not adequately engaged in policy communication processes and strategies neither did they provide feedback regarding the policy performance. A study done in Ghana supports this finding by stating that when HCWs and users understand the primary intentions of a health policy change they gain ownership and became the ambassadors of policy change dissemination [14]. This is done through empowering them with knowledge and early involvement in policy processes including formulating communication strategies [14]. However; this is contrary with the study done in USA which highlighted that over consultations with the grass root could derail progress especially when dealing with a magnitude of a public health problem such as HIV/AIDS that requires urgency [24].

The test and treat policy changes were abruptly introduced in Zambia and most of the implementers were caught unaware. Immediately the policy change was pronounced, the policy makers, partners and politicians started to actively champion it through adverts on various media platforms, community meetings and road shows. Unfortunately, the key messages were not tallying with services in health facilities in terms of infrastructure, practice, human and material 
resources on the ground. This brought confusion on how best to implement the changes. The frontline HCWs experienced task shifting, and felt demotivated due to increased workload to accommodate the policy change. This finding is in line with a qualitative study done in Uganda where an HIV program implementation was introduced haphazardly without proper communication strategies to implementers and investing in human and material resources [25]. This jeopardized HIV service delivery due to inadequate resources and capacity the program [25]. Another study done by Lo-Beer et al found that inadequate HIV policy change communication directives can bring confusion to existing health services and delivery if not communicate well due to imprecise changes to be adopted [26].

We found that the test and treat policy was misinterpreted as a mandatory HIV test by many HCWs to all clients who visited health facilities for any ailment due deficient information on service provision and protocols. However, not all clients accepted this new change of HIV service provision as the benefits were not emphasized. This is in compliment with other studies done around the globe which highlighted that in order to have successful policy implementation there is need to pilot new health policies changes to allow diffusion and enhance uptake [27, 28]. Health policy change uptake can be enhanced if the implementer and user understood what was supposed to be done [29]. Many shunned health facilities in preference for over-the-counter medications defeating worthiness of the HIV policy change intentions. This could result in high HIV incidence rate.

The study findings further revealed that there were no documented formal HIV policy communication protocols in all health facilities with everything left to the discretion of policy makers and tertiary health facility managers or senior management in health facilities. Lack of communication protocols lead to confusion in policy directives on the ground. This is in line with the study done by Gilson who suggested policy directives should be documented and mentored continuously for best outcomes [16]. Additionally, policy makers, partners and NAC managers did not collaborate with health facility managers, frontline HCWs and the public to communicate policy change for successful implementation. They presumed their official channels of communication were effective contrary to what was obtaining on the ground. Studies have shown that working with the grassroots and providing policy directive frameworks for HIV management accelerates uptake of policy changes [30, 31].

To communicate test and treat HIV health policy change, government relied on print media like newspapers, posters and brochures; electronic media such as public and private radios, television and social media pages like Facebook, WhatsApp and MoH websites which were ineffective. Print media such as newspapers both for public and private had inadequate impact to communicate the policy. Though newspapers could be stored for reference and exchanged amongst the public literacy levels were said to be very low to be appreciated by many clients and mostly accessed along the line of rail. This is in line with a study done in Tanzania which showed that HCWs even given protocols can be poorly utilized due to poor reading cultures [32]. The television sets and radios were not common gadgets to access information from and expensive despite having a nationwide coverage including rural areas. There were reported power outages in the city that denied access to information from televisions and radios.

Social media had a wider coverage but mainly accessed by privileged youths in urban areas and people with white collar jobs. Due to different opinions on the test and treat policy, debates were generated for and against the new services the various media platforms. The HCWs had The MoH and partners printed plenty of information education and communication (IEC) materials with test and treat HIV policy change key messages on posters and brochures to support information dissemination to HCWs and the public. Studies done in USA suggest that HIV health policy literacy is important to avert barriers to information [33].The public and implementers must be incorporated to design health policy communicating strategies to maximize policy uptake with open feedback mechanisms [34]. Physical discussions on HIV policy change were often held in health facilities. This gave chance to patients to clarify on the policy.

The test and treat HIV policy was implemented differently across health facilities. Some HCWs provided some counselling to clients while others did not negotiate and made anti-retroviral treatment compulsory for newly HIV diagnosed. This is contrary to studies done in in Zambia that revealed that consent to HIV testing and management is still a fundamental human right that should be treated with autonomy for every individual or else may negate good intention of HIV services if coerced. Therefore, there is a need to enhance awareness and supervise the health facilities so that the implementation of the HIV test and treat health policy change is not left to the discretion of frontline HCWs.

Given the above findings, the gap of inadequate communication of test and treat HIV health policy change if not addressed could derail policy change uptake into 2030. The dream for elimination of HIV may not be achieved. Therefore, there is a need to provide test and treat policy communication guidelines and protocols to all health facilities for standard practice.

\section{Study strengths and limitations}

The selection of participants interviewed had varied occupations in the healthcare systems and credible data was collected due to variations in function and competencies; while health facility level ranged from primary, secondary to tertiary level of care which enhanced data validity and quality [35]. This consolidated content and face validity was achieved. The data was deemed reliable as answers become more consistent across all related respondents [36]. Most of the participants though holding the targeted offices had served for less time to understand the test and treat HIV health policy as Ministry was undergoing restructuring; while many participants were newly employed. The cost following up participants was an issue due to the outbreak of COVID-19 pandemic as many HCWs were restricted to contact visitors and rescheduled appointments quiet often. Time for KII and FGDs was reduced to no more than an hour due to COVID 19 for observing social distances, rescheduled working hours and staying home rules. So smaller FDGs were opted for safety.

\section{Conclusion}

The study showed that the involvement of implementers in test and treat HIV health policy change communication processes was not prioritized by government, which affected implementation and policy ownership among the HCWs. The media adverts for the test and treat HIV policy change should have should been designed in collaboration policy makers, partners, implementers and users to match with information on the ground especially relating to practice 
to enhance policy uptake. Further, we find that it was cardinal to empower HCWs with trainings to understand policy change guidelines, expected outcomes and impact from the test and treat HIV health policy change.

The hallmark of the adoption of test and treat HIV policy was to eliminate HIV/AIDS in Zambia by the year 2030. The status of the findings on the communication and implementation revealed that the dream was far from being realized while the goal to control HIV by 2020 was threatened by the outbreak of the corona virus pandemic. There was need to do more on the collaborations amongst stakeholders; distillation of information; standard communication processes and strategies for all to buy in and practice the test and treat HIV health policy change uniformly with enhanced feedback loops and mechanisms while addressing unexpected effects initiated in the health systems.

\section{Abbreviations}

HIV

Human Immunodeficiency Virus

HCWs

Health Care Workers

T\&T

Test and Treat

NAC

National HIV/AIDS/STI/TB Council

$\mathrm{MoH}$

Ministry of Health

\section{Declarations}

\section{Acknowledgments}

This work would not have been finalized without the support and contribution of the supervisory team of lecturers from the University of Zambia namely Dr. Selestine Nzala, Dr. Halwiindi Hikabasa and Mr Adam Silumbwe. We further would like to thank all the health facilities and staff in Lusaka District where data was collected, Ministry of Health policy makers, partners and National HIV/AIDS/STI/TB council managers for according us time from their busy schedules.

\section{Author's contribution}

CS, AS, and SN conceived the study. CS did the data collection; AS, SN and HH provided supervision and support during data collection. CS, AS, SN and JMZ did the analysis and drafted the manuscript arrangement. AS, SN, HH, JMZ edited and reviewed various versions of the manuscript. AS, SN and HH oversaw and guided the entire writing process. All authors read and approved the final manuscript.

\section{Funding}

The University of Zambia provided financial support for the academic process of first author. The family and friends played a key role.

\section{Availability of data and materials}

The field notes and interview recorded verbatim and transcripts generated during data collection and analysis of this study are not publicly available to ensure the confidentiality and anonymity of the participating organizations and participants but can be accessed on request.

The authors alone are responsible for the views expressed in this article and they do not necessarily represent the opinions, decisions, or policies of the institutions to which they are affiliated.

\section{Ethics approval and consent to participate}

Ethical approval was provided by the UNZABREC [reference number 277-2019] and clearance to conduct research was sought and granted by the NHRA. Written informed consent was sought from individual participants before data collection.

\section{Competing Interest}

The authors declare that they have no competing interests.

\section{Consent for publication}

Not applicable

\section{Author's information}

At the time of drafting this manuscript, the first author: Constance Simooya was a student in the Department of Health Policy and Management at the University of Zambia. This manuscript is part of a Master's thesis on Health policy and implementation into the health systems in Lusaka District of Zambia. 


\section{References}

1. UNAIDS, J.U.N.P.o.H A, Global report: UNAIDS report on the global AIDS epidemic 2012. 2012. URL: http://www. unaids. org/ sites/ default/ files/ media_asset/ 20121120_UNAIDS_Global_Report_2012_with_annexes_en_1. pdf [accessed 2017-01-30][WebCite Cache], 2018.

2. Rajasingham R, et al. Global burden of disease of HIV-associated cryptococcal meningitis: an updated analysis. Lancet Infect Dis. 2017;17(8):873-81.

3. Chanda-Kapata P, et al. The adult prevalence of HIV in Zambia: results from a population based mobile testing survey conducted in 2013-2014. AIDS Res therapy. 2016;13(1):1-9.

4. Nakazwe C, et al. Contrasting HIV prevalence trends among young women and men in Zambia in the past 12 years: data from demographic and health surveys 2002-2014. BMC Infect Dis. 2019;19(1):1-9.

5. Nzali Kancheya M, et al., Increase in Antiretroviral Therapy Enrollment Among Persons with HIV Infection During the Lusaka HIV Treatment Surge-Lusaka Province, Zambia, January 2018-June 2019.

6. NAC, N.H.A.S.T.C.. Zambia Legal environment for HIV, TB, and sexual and reproductive health rights 2020. [cited Available from.

7. Gage AJ, Know Your HIV-Prevention, Response. Southern Province, Zambia. Chart Book on Implementation of HIV-prevention Intervenions by Nongovernmental Organizations. USAID: Chapel Hill; 2014.

8. Hanunka B, et al., Effectiveness of Option B+ in reducing mother-to-child transmission of HIV: A retrospective cohort study of pregnant women in 6 public health facilities in Lusaka, Zambia. 2018.

9. MoH MoH-. Zambia CONSOLIDATED GUIDELINES for prevention and treatment of HIV infection 2018. [cited Available from:

https://www.nac.org.zm/sites/default/files/publications/Zambia\%20Consolidated\%20Guidelines\%20for\%20HIV\%20Treatment\%20and\%20Prevention_20

10. Munthali T, et al. Impact of WHO guidelines on trends in HIV testing and ART initiation among children living with HIV in Zambia. AIDS Res therapy. 2020;17:1-10.

11. Cawley $\mathrm{C}$, et al. Identifying gaps in HIV policy and practice along the HIV care continuum: evidence from a national policy review and health facility surveys in urban and rural Kenya. Health Policy Plann. 2017;32(9):1316-26.

12. Mesic A, et al. Facilitators and barriers to adherence to antiretroviral therapy and retention in care among adolescents living with HIV/AIDS in Zambia: A mixed methods study. AIDS Behav. 2019;23(9):2618-28.

13. Qiao S, et al. Facilitators and barriers for HIV-testing in Zambia: a systematic review of multi-level factors. PLoS ONE. 2018;13(2):e0192327.

14. Koduah A, van Dijk H, Agyepong IA. The role of policy actors and contextual factors in policy agenda setting and formulation: maternal fee exemption policies in Ghana over four and a half decades. Health Res policy Syst. 2015;13(1):27.

15. Romanenko YO. Place and role of communication in public policy. Актуальні проблеми економіки, 2016(2): p. 25-31.

16. Gilson L. Everyday politics and the leadership of health policy implementation. Health Syst Reform. 2016;2(3):187-93.

17. Gilson L, et al. Everyday resilience in district health systems: emerging insights from the front lines in Kenya and South Africa. BMJ Global Health. 2017;2(2):e000224.

18. Paladino J, Lakin JR, Sanders JJ. Communication strategies for sharing prognostic information with patients: beyond survival statistics. JAMA. 2019;322(14):1345-6.

19. Merriam SB. Case study research in education: A qualitative approach. Jossey-Bass. [cited Available from;; 1988.

20. Boyd MA, et al. Increase in Antiretroviral Therapy Enrollment Among Persons with HIV Infection During the Lusaka HIV Treatment Surge-Lusaka Province, Zambia, January 2018-June 2019. Morb Mortal Wkly Rep. 2020;69(31):1039.

21. Gale NK, et al. Using the framework method for the analysis of qualitative data in multi-disciplinary health research. BMC Med Res Methodol. 2013;13(1):1-8.

22. Thompson TL, Parrott R, Nussbaum JF. The Routledge handbook of health communication. Routledge; 2011. [cited Available from;

23. Sutton J, Austin Z. Qualitative research: Data collection, analysis, and management. Can J Hosp Pharm. 2015;68(3):226.

24. Anderson S, et al. Perspectives of HIV clinic staff on the implementation of a client financial incentives program targeting viral suppression. $J$ Assoc Nurses AIDS Care. 2017;28(5):770-83.

25. Zakumumpa H, Bennett S, Ssengooba F. Modifications to ART service delivery models by health facilities in Uganda in promotion of intervention sustainability: a mixed methods study. Implement Sci. 2017;12(1):1-14.

26. Low-Beer D, Stoneburner RL. Behaviour and communication change in reducing HIV: is Uganda unique? Afr J AIDS Res. $2003 ; 2(1): 9-21$.

27. Nilsen P, et al. Implementation of change in health care in Sweden: a qualitative study of professionals' change responses. Implement Sci. $2019 ; 14(1): 51$.

28. Lewinski AA, Simmons LA. Nurse knowledge and engagement in health policy making: Findings from a pilot study. J Continuing Educ Nurs. 2018;49(9):407-15.

29. Semitala FC, et al., Understanding uptake of an intervention to accelerate antiretroviral therapy initiation in Uganda via qualitative inquiry. J Int AIDS Soc, 2017. 20(4).

30. Babalola S, et al, A framework for health communication across the HIV treatment continuum. Journal of acquired immune deficiency syndromes (1999), 2017. 74(Suppl 1): p. S5.

31. Ansell C, Sørensen E, Torfing J. Improving policy implementation through collaborative policymaking. Policy \& Politics. 2017;45(3):467-86.

32. Shayo $\mathrm{EH}$, et al. Challenges of disseminating clinical practice guidelines in a weak health system: the case of HIV and infant feeding recommendations in Tanzania. Int Breastfeed J. 2014;9(1):1-13. 
33. Kalichman SC, et al., Health literacy and health-related knowledge among persons living with HIV/AIDS. American journal of preventive medicine, 2000. 18(4): p. 325-331.

34. Pierson P. When effect becomes cause: Policy feedback and political change. World Polit. 1993;45(4):595-628.

35. Guba EG, Lincoln YS. Competing paradigms in qualitative research. Handb qualitative Res. 1994;2(163-194):105.

36. Mallinson S. Listening to respondents:: a qualitative assessment of the Short-Form 36 Health Status Questionnaire. Soc Sci Med. 2002;54(1):11-21.

\section{Tables}

Table 1: Summary of key-informant interviews

\begin{tabular}{|lll|}
\hline Participants & Number & Institution \\
\hline Policy Makers - Health policy department & 4 & $\mathrm{MoH}$ \\
\hline Policy Makers - Public health clinical & 3 & $\mathrm{MoH}$ \\
\hline Program manager HIV partners & 1 & PEPFAR \\
\hline National HIV/AIDS.STI/TB Council Managers & 3 & NAC \\
\hline Health Facility Managers & 2 & Tertiary health facility \\
\hline District Health Office Facility Managers & 1 & LDHO \\
\hline District Health Office HIV/AIDS program Managers & 2 & LDHO \\
\hline Health Facility Managers - clinical & 3 & Secondary health facility \\
\hline Health facility manager & 3 & Primary health care \\
\hline Total & $\mathbf{2 2}$ & \\
\hline
\end{tabular}

Table 2: Summary of focus group discussions with frontline HCWs

\begin{tabular}{|c|c|c|c|c|}
\hline \multicolumn{2}{|c|}{ Level of healthcare facility } & \multicolumn{2}{|l|}{ Participant category } & \multirow{2}{*}{$\begin{array}{l}\text { Total } \\
8\end{array}$} \\
\hline Tertiary & 1 & Doctors & 1 & \\
\hline & & Nurses & 5 & \\
\hline & & Psychosocial counsellors & 2 & \\
\hline \multirow[t]{3}{*}{ Secondary } & 1 & Doctors & 1 & 11 \\
\hline & & Nurses & 7 & \\
\hline & & Psychosocial counsellors & 3 & \\
\hline \multirow[t]{2}{*}{ Primary } & 1 & Nurses & 5 & 7 \\
\hline & & Psychosocial counsellors & 2 & \\
\hline Total & 3 & & & 26 \\
\hline
\end{tabular}

Table 3: Qualitative data code-list 


\begin{tabular}{|c|c|}
\hline Major theme categories & Sub-theme categories \\
\hline \multirow[t]{4}{*}{ 1. Communication of test and treat HIV health policy change } & $\begin{array}{l}\text { - Communication strategy for HIV/AIDS policy change } \\
\text { - HIV/AIDS strategic framework for } 2017 \text { to } 2021\end{array}$ \\
\hline & - Communication channels of policy change \\
\hline & $\begin{array}{l}\text { - The role of the media in communication policy change to test-and-treat-all } \\
\text { - National television and radio stations }\end{array}$ \\
\hline & - Information education and communication, and other print media (online media) \\
\hline \multirow[t]{5}{*}{ 2. Implementation of test and treat HIV policy change } & - Historical context and knowledge of HIV/AIDS health policy changes \\
\hline & - Stakeholder engagement in test and treat health policy change \\
\hline & - Healthcare worker trainings for implementation of test and treat policy \\
\hline & - Materials and resources to support test and treat HIV health policy change \\
\hline & - Financing test and treat HIV health policy change \\
\hline \multirow{2}{*}{$\begin{array}{l}\text { 3. Changes brought about by the HIV policy change to test-and- } \\
\text { treat }\end{array}$} & - Human resources and infrastructure adjustment \\
\hline & - Organisational change HIV service delivery \\
\hline \multirow[t]{3}{*}{ 4. Acceptability of test and treat HIV policy change } & - Accepatability of policy change among health providers \\
\hline & - Sense of ownership of policy change \\
\hline & - Policy change resistance by community members \\
\hline
\end{tabular}

\section{Supplementary Files}

This is a list of supplementary files associated with this preprint. Click to download.

- AdditionalfileCOREQChecklist.pdf 